\title{
Experiments on intrinsic and thermally induced chaos in an rf-driven Josephson junction
}

Davidson, A.; Dueholm, B.; Beasley, M. R.

\section{Published in:}

Physical Review B

Link to article, DOI:

10.1103/PhysRevB.33.5127

Publication date:

1986

Document Version

Publisher's PDF, also known as Version of record

Link back to DTU Orbit

Citation (APA):

Davidson, A., Dueholm, B., \& Beasley, M. R. (1986). Experiments on intrinsic and thermally induced chaos in an rf-driven Josephson junction. Physical Review B, 33(7), 5127-5130. https://doi.org/10.1103/PhysRevB.33.5127

\section{General rights}

Copyright and moral rights for the publications made accessible in the public portal are retained by the authors and/or other copyright owners and it is a condition of accessing publications that users recognise and abide by the legal requirements associated with these rights.

- Users may download and print one copy of any publication from the public portal for the purpose of private study or research.

- You may not further distribute the material or use it for any profit-making activity or commercial gain

- You may freely distribute the URL identifying the publication in the public portal 


\title{
Experiments on intrinsic and thermally induced chaos in an rf-driven Josephson junction
}

\author{
A. Davidson \\ IBM Research Center, P.O. Box 218, Yorktown Heights, New York 10598 \\ B. Dueholm \\ Physics Laboratory I, Technical University of Denmark, DK2800 Lyngby, Denmark \\ M. R. Beasley \\ Department of Applied Physics, Stanford University, Stanford, California 94305
}

(Received 3 February 1986)

\begin{abstract}
We report detailed measurements of low-frequency noise due to microwaves applied to a real Josephson tunnel junction. An intrinsically chaotic region is apparently identified, but the effects of thermal noise are shown to be significant. In particular we show experimental data that we interpret as evidence for thermally activated hopping and thermally affected chaos. The data are only in qualitative accord with recent ideas regarding the effect of thermal noise on intermittent chaos.
\end{abstract}

Huberman, Crutchfield, and Packard ${ }^{1}$ first showed that the forced-pendulum equation admitted chaotic solutions in 1980. Since then the literature has steadily grown in the study of these solutions by simulations ${ }^{2-10}$ and analysis. ${ }^{11}$ However, although the forced pendulum corresponds to many interesting and important systems, such as synchronous motors, phase-locked-loop radio receivers, pinned charge-density waves, and Josephson junctions, experimental work in these other systems, save in phase-locked-loop Josephson analogs, has lagged far behind. Yet real systems have some advantages over simulations that make them important to study quite apart from using them to confirm simulation results. Josephson junctions in particular offer very-high-speed dynamics that make the precise measurement of low-frequency mean values practical (one second of integration can average over billons of Josephson periods, so that very unlikely events can still be detected in practical times). Further, Josephson junctions can be produced with characteristic energies close to thermal values, so that the interaction of thermal noise with chaos is experimentally accessible. Finally, the correspondence of the Josephson dynamical system to a forced pendulum is among the cleanest and best documented. Despite the attractions, only limited studies on real Josephson junctions have been reported. ${ }^{12-16}$

We report results of detailed experiments on the excess noise that occurs between Josephson steps for conditions that are thought to be appropriate for intermittent chaos. We observe a significant thermal influence, which, depending on bias current, appears to be thermally induced hopping or thermally affected intrinsic (chaotic) intermittency. 9 , 10 Since for our junctions the ratio of the thermal energy to the Josephson coupling energy $k_{B} T / E_{J} \cong 10^{-3}$, our results demonstrate the extreme sensitivity of chaos to thermal influences at least under the present circumstances.

The Josephson tunnel junctions we studied were made with a $\mathrm{Nb}$ base and $\mathrm{Pb}$ alloy counterelectrodes, with the tunnel barrier formed of $\mathrm{Nb}_{2} \mathrm{O}_{5}$ on an edge of the $\mathrm{Nb}$ film. ${ }^{17}$ The junction was made smaller than a Josephson penetration depth and had a plasma frequency of about $150 \mathrm{GHz}$. To lower this frequency into a more convenient range, the junction was made on a portion of the edge of a square capacitor, about $80 \times 80 \mu \mathrm{m}^{2}$, with about $400 \AA$ of $\mathrm{Nb}_{2} \mathrm{O}_{5}$ as the dielectric ( $C \cong 15 \mathrm{pf}$ ). A $1.5-\Omega$ resistive shunt was also incorporated, so that the final result was a junction with about $40 \mu \mathrm{A}$ of critical current, roughly a $14-\mathrm{GHz}$ plasma frequency, and a $\beta_{c}=Q^{2}$ of about 4 . The entire circuit was made over a $\mathrm{Nb}$ ground plane and attached to various microwave coupling structures in a cryogenic microwave box using $50-\Omega$ coaxial-line-to-strip-line launchers. The junc-
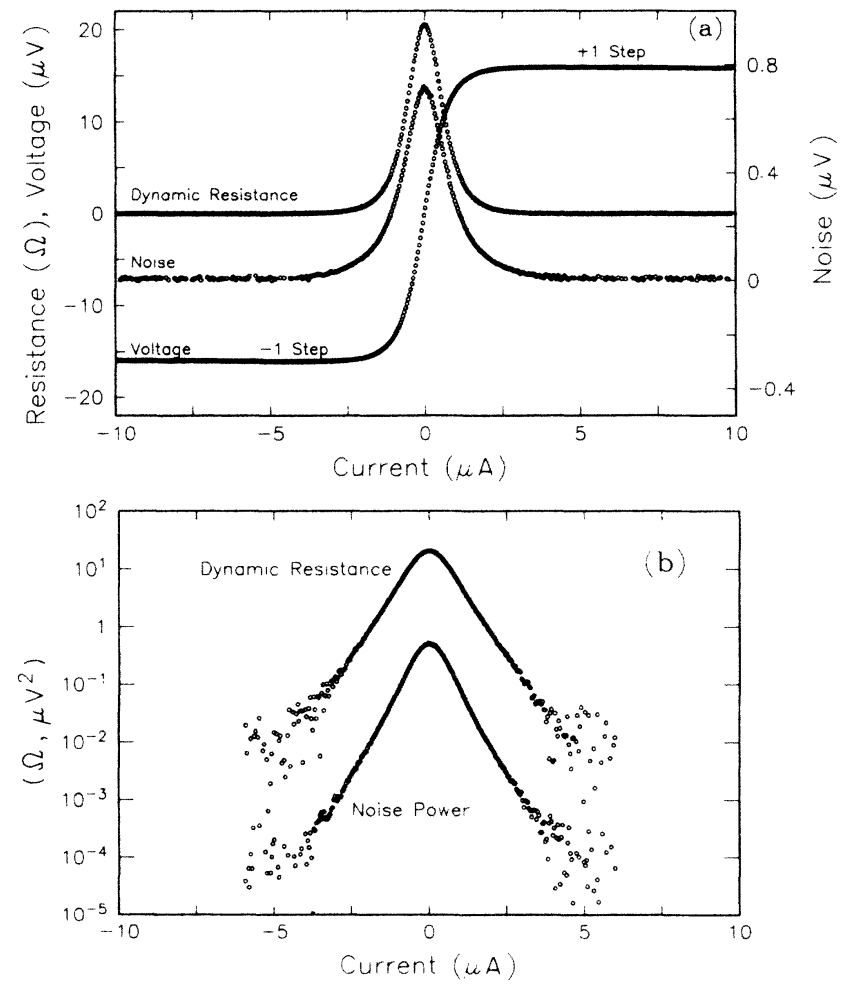

FIG. 1. $I-V$ curve, dynamic resistance, and noise as measured along a transition between the $n=-1$ and the $n=+1$ steps for a junction with parameters as described in the text. The semilog graph in (b) shows the exponential character of the noise and resistance. 
tion displayed a canonical resistively shunted-junction-model $I-V$ characteristic, with the expected amount of hysteretic switching to and from a smooth finite voltage curve.

Figure 1(a) shows the voltage, dynamic resistance, and voltage noise for one particular setting of the $\mathrm{rf}$ attenuation and an rf frequency of $8.1 \mathrm{GHz}$. Generally, every transition between steps on the $I-V$ curve was accompanied by measureable noise for particular if power levels. For simplicity we have focused our measurements on the region near zero bias current.

For the data presented here, we restrict our measurements further by keeping the rf power level within about one decibel of where the supercurrent (i.e., where the $n=0$ Josephson step) first disappears, and where there appear only the two steps on either side of zero current (the -1 and +1 rf Josephson steps). It is known from the whole body of simulations in the literature that this is the threshold for intermittent chaos. At higher rf attenuation the $I-V$ curve varied smoothly from the -1 step through the $n=0$ step to the +1 step, with little noise. At lower values of attenuation there was again little noise as the $I-V$ curve became hysteretic, with the +1 and -1 steps overlapping around zero bias. In between these two regions the transition between the two steps became sharper as the attenuation was reduced, but, as in Fig. 1, it remained smooth with more and more noise until the point where hysteresis set in. The largest noise was always centered at zero-bias current. This behavior is in good agreement with simulations ${ }^{3}$ that show intermittent chaos in a narrow range of $\mathrm{rf}$ power at zero-bias current, just where the $n=0$ Josephson step (the supercurrent) disappears.

Figure 1(b) shows a semilog plot of the noise and dynamic resistance for the data in Fig. 1(a). Both measurements were made using a Princeton Applied Research No. 124 lock-in amplifier with a low-noise preamp $\left(1 \mathrm{nV} / \mathrm{Hz}^{1 / 2}\right)$. The dynamic resistance was taken in the usual lock-in derivative technique, with an excitation frequency and amplitude of $900 \mathrm{~Hz}$ and $100 \mathrm{nA}$, rms. The noise was measured with the lock-in in the ac-voltmeter mode at $90 \mathrm{kHz}$, $10 \%$ equivalent noise bandwidth. Both signals were averaged using digital techniques on a microcomputer. Using this system we could see noise below the amplifier's limit by digitally subtracting the measured amplifier noise in the square. The practical limit to our sensitivity was about 0.1 $\mathrm{nA} / \mathrm{Hz}^{1 / 2}$. It may be observed from Fig. 1(b) that the noise power and dynamic resistance are roughly proportional (within a factor of 3 ) and have an exponential rise from the noise background, a feature common to all our data. Using the dynamic resistance to define an effective noise temperature, for these particular data we find $T_{N} \cong 50000 \mathrm{~K}$ over the entire range above the instrument background. It should also be noted that in the exponential region of the curve, the noise power must be also proportional to the voltage deviation from the nearest step. This proportionality is consistent with Poisson (shot-noise-like) statistics in the noise mechanism: The noise voltage consists of discrete bursts of about the same size occurring at random. The magnitude of the bursts, or the size of the phase slip, may be inferred from the shot-noise formula $n \phi_{0}=V_{n}^{2} / 2 V_{\mathrm{dc}}$. Our data show $n$ to be typically in the range from 50 to 200 .

Figure 2 is a semilog plot that shows the effect of adding external noise into the microwave coaxial line feeding the junction. The wide-band noise source was estimated to double the external thermal noise, ${ }^{18}$ driving the junction

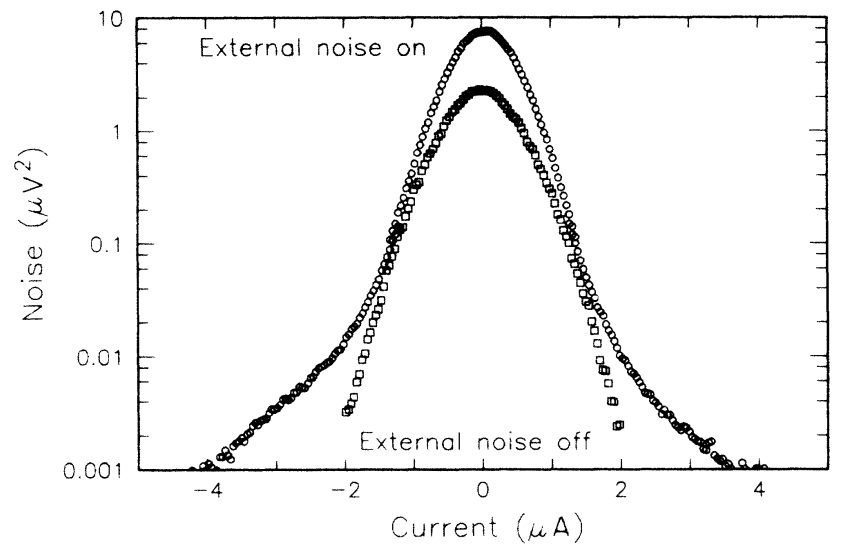

FIG. 2. Semilog plot of noise power for an rf attenuation different from Fig. 1. This plot shows a dramatic shift in amplitude and width when a roughly $600-\mathrm{K}$ external noise source is turned on. Note that the largest change is in the exponential wings at large dc bias.

over a broad bandwidth from a few $\mathrm{MHz}$ to $18 \mathrm{GHz}$. There was no attenuation added to the coaxial line between the junction in liquid helium and the room-temperature sources for this part of the experiment. This extra noise is observed to increase the measured noise amplitude, and distinct lownoise wings appear for currents well away from zero. These wings are also faintly evident in Fig. 1(b) for noise levels below $10^{-2}(\mu \mathrm{V})^{2}$. Figure 2 shows conclusively that external noise can have a profound influence on the noise level, most particularly at the transitions at the ends of the steps.

As also seen in Fig. 2, the amplitude of the noise has different functional forms in the two regions: an exponential rise from the background, and a more parabolic (Gaussian) central peak. In fact, we have found empirically that curves of the form

$$
V_{n}^{2}=A e^{-I / I_{0}}+B e^{-I^{2} / I_{1}^{2}}
$$

fit all our data very well. As a test we measured 16 detailed noise curves at 4.2 and $3.06 \mathrm{~K}$, using cold rf attenuators to minimize the influence of $300-\mathrm{K}$ noise, with increments of $0.1 \mathrm{~dB}$ between 29.6 and $28.9 \mathrm{~dB}$. Two typical such curves out of the 16 are shown in Fig. 3, together with the fits of Eq. (1). $A, B, I_{0}$, and $I_{1}$ were all free fitting parameters. The fits to the other 14 curves were of similar quality. The dependence on rf attenuation of the four fitting parameters is shown in Fig. 3(b) for the 8 curves taken at $3.06 \mathrm{~K}$. The data at $4.2 \mathrm{~K}$ were very similar, with an increase in $I_{1}$ and $I_{0}$ by a factor of 1.2 .

How can these data (for which no explicit prediction exists) be interpreted in the light of current ideas of noise and chaos in the forced pendulum? These issues have been investigated through simulations most recently by Westervelt and Gwinn ${ }^{9}$ and by Kautz. ${ }^{10}$ These authors stress the distinction between thermally induced hopping or extrinsic intermittency (between states that may themselves be stable or chaotic at $T=0$ ) and thermally affected chaos in which a state that is intrinsically chaotic at $T=0$ is slightly influenced by the addition of thermal noise. Our data might reflect just these two regimes, depending on bias current. Our decomposition of the current dependence of the noise power into an exponential and a Gaussian portion could already be a rough separation into these two processes, as dis- 

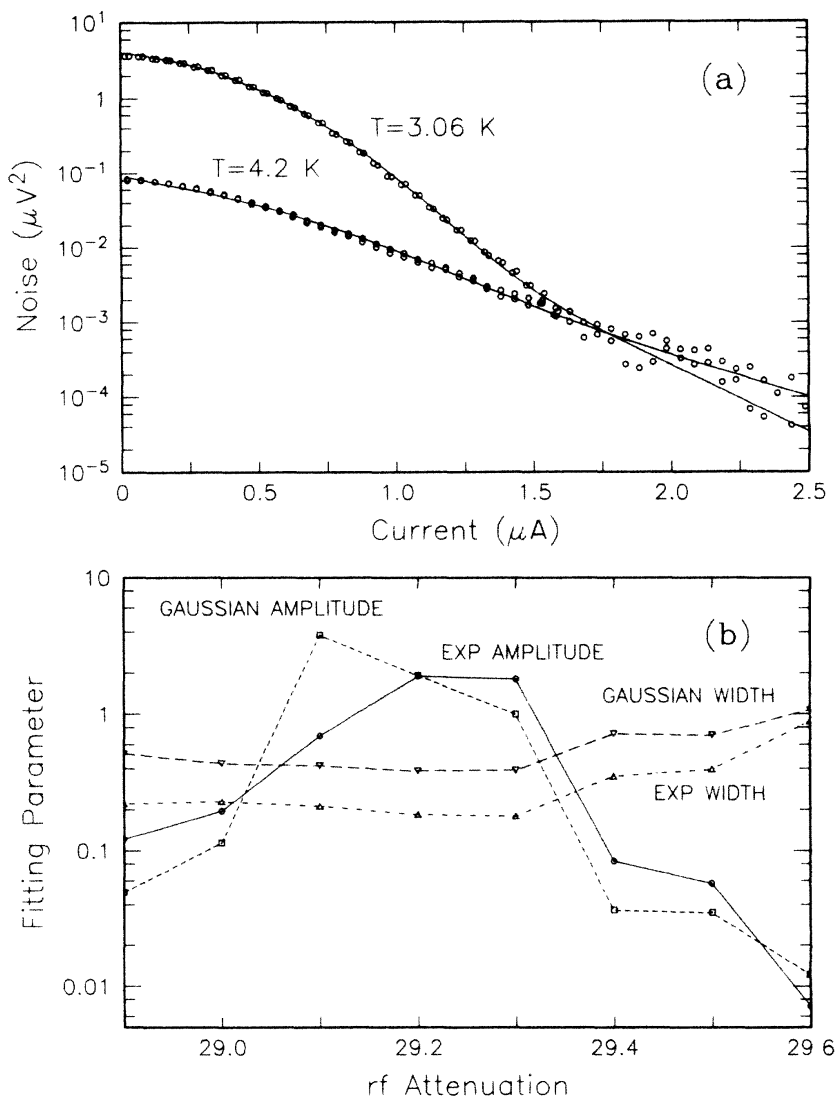

FIG. 3. (a) Four parameter fits of Eq. (1) to two examples of the 16 noise curves taken at 4.2 and $3.06 \mathrm{~K}$. The junction had the same parameters as before, but the microwave frequency was $7.5 \mathrm{GHz}$. (b) Variation of the four fitting parameters from Eq. (1) with microwave attenuation for the 8 curves taken at $3.06 \mathrm{~K}$. Below 28.9$\mathrm{dB}$ attenuation the junction became visibly hysteretic and essentially noiseless. Above $29.6 \mathrm{~dB}$ there was evidence of a small supercurrent, and no measurable noise.

cussed below.

The systematics of our data may be visualized in terms of the schematic state diagram in Fig. 4, which is a map in the rf-power $(\gamma)$-bias-current plane. based on simulation results. $^{3}$ The crosshatched region corresponds to intrinsic intermittent chaos and the clear regions correspond to stable rf Josephson steps. As shown in Ref. 3, such steps can be chaotic with a noise spectrum that is large at high frequencies, but which goes to zero at zero frequency. The data presented here correspond to the region between $\gamma_{c 1}$ and $\gamma_{c 2}$. The issue addressed by our experiments is the nature of the transitions between the stable and intermittent regions. The transition from a region of hysteretic, symmetry-broken running states of the forced pendulum has been discussed recently by Gwinn and Westervelt (GW). ${ }^{9}$ Their analysis deals with the transition as a function of rf level $(\gamma)$, at zero-bias current. They find that at the transition the system hops between the two previously stable states $(n=+1$ and -1 in our case) at a rate $1 / \tau \propto\left(\gamma_{c}-\gamma\right)^{1 / 2}$ where $\gamma_{c}=\gamma_{c 1}$ is the critical onset value of $\gamma$. Kautz $\mathrm{z}^{10}$ has considered a similar situation and successfully modeled the noise as a random telegrapher signal. When the two states have the same mean lifetimes (as they shoud by symmetry at zero bias) he finds $V_{n}^{2}(0) \propto \tau$. Com-

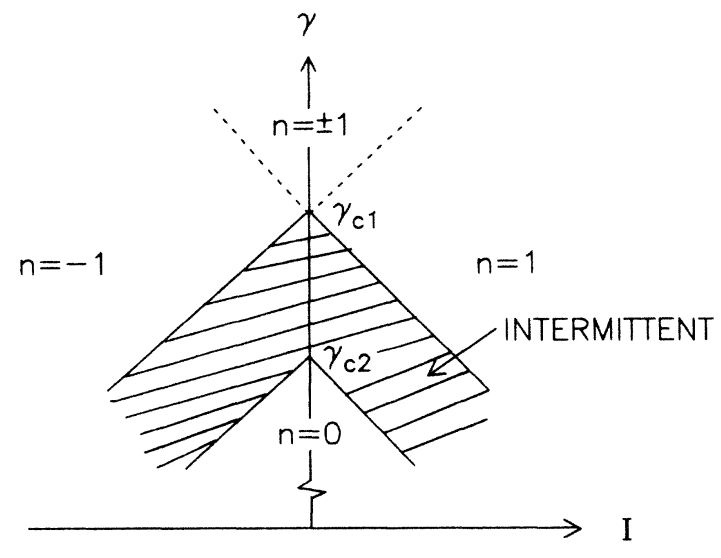

FIG. 4. Schematic diagram for the chaotic region studied in these experiments. $\gamma$ is the rf-power level, and $I$ the bias current. Note that the $n= \pm 1$ region at the top is bistable and hysteretic.

bining this result with that of $\mathrm{GW}$ one obtains $V_{n}^{2}(0)$ $\propto\left(\gamma_{c 1}-\gamma\right)^{-1 / 2}$ just beyond the transition. Thus, an abrupt rise in the noise followed by a gradual falloff would be expected.

As seen in Fig. 3(b) our data show an abrupt rise in the Gaussian noise power in the vicinity of $29.0 \mathrm{~dB}\left(\gamma=\gamma_{c 1}\right)$. The Gaussian component then slowly declines as the attenuation is further increased ( $\gamma$ decreased), in qualitative agreement with these simple theoretical considerations. Breaking symmetry with a bias current favors intermittent hopping to one state over the other and reduces the noise. If the differences in the mean switching times increase in proportion to $I$, the random telegrapher noise model predicts $V_{n}^{2}(0)$ will decrease proportionally to $I^{2}$. This agrees with our Gaussian fitting function, but there is no obvious reason in this picture why $I_{1}$ should be nearly constant. Also, since $I_{1}$ is temperature dependent, some thermal influence is indicated: For example, $I_{1}$ could be related to the rms amplitude of the noise that accompanies the rf bias.

As regards the transition into the intermittent region from the $n=+1$ and $n=-1$ states as a function of bias current, our data support the idea that this process is shotlike. Since the $I-V$ curve is exponential, thermally activated shot noise is suggested. The simplest assumption is that the exponent is determined by the work done by the external current source during a phase slip, which would be the order of $\phi_{0} I$, which corresponds to $I_{0}=2 k T / \phi_{0}$, where $\phi_{0}=h / 2 e$ is the flux quantum. For $T=3.06 \mathrm{~K}$ this yields $I_{0} \cong 0.06 \mu \mathrm{A}$, only a factor of 4 below our exponential fitting width.

We wish to thank N. F. Pedersen for his encouragement of this experiment, and for useful discussions. We also acknowledge useful discussions with D. J. Frank, D. D'Humieres, Y. Imry, and M. Buettiker. Our junctions were made in a process developed by a group including K. H. Brown, T. Bucelot, A. C. Callegari, M. M. Chen, J. H. Greiner, H. C. Jones, K. K. Kim, A. W. Kleinsasser, H. A. Notarys, G. Proto, R. H. Wang, and T. Yogi. M. J. Brady made the low-inductance microwave structures on which the chips were mounted. Work at Stanford was supported by the National Science Foundation, Department of Materials Research. 
${ }^{1}$ B. A. Huberman, J. P. Cruthchfield, and N. H. Packard, Appl. Phys. Lett. 37, 750 (1980).

${ }^{2}$ N. F. Pedersen and A. Davidson, Appl. Phys. Lett. 39, 830 (1981).

${ }^{3}$ D. D'Humieres, M. R. Beasley, B. A. Huberman, and A. Libchaber, Phys. Rev. A 26, 3483 (1982).

${ }^{4}$ I. Goldhirsh, Y. Imry, G. Wasserman, and E. Ben-Jacob, Phys. Rev. B 29, 1218 (1984).

${ }^{5}$ R. F. Miracky and J. Clarke, Appl. Phys. Lett. 43, 508 (1983).

${ }^{6}$ R. L. Kautz, IEEE Trans. Magn. MAG-19, 465 (1983).

${ }^{7}$ R. L. Kautz and R. Monaco, J. Appl. Phys. 57, 875 (1985).

${ }^{8}$ H. Seifert, Phys. Lett. 101A, 230 (1984).

${ }^{9}$ E. G. Gwinn and R. M. Westervelt, Phys. Rev. Lett. 54, 1613 (1985).

10R. L. Kautz, J. Appl. Phys. 58, 424 (1985).

${ }^{11}$ Z. H. Genchev, Z. G. Ivanov, and B. N. Todorov, IEEE Trans. Circuits Syst. CAS-30, 633 (1984); F. M. A. Salam and S. S. Sastry, ibid. (to be published); M. Bartuccelli, P. L. Christensen, N. F. Pedersen, and M. T. Sorensen (private communication).

${ }^{12}$ V. N. Gubankov, K. I. Konstantinyan, V. P. Koshelets, and G. A. Ovsyannikov, IEEE Trans. Magn. MAG-19, 637 (1983).
${ }^{13} \mathrm{C}$. Readi Nasser and M. Octavio, in Proceedings of the Seventeenth International Conference on Low Temperature Physics, edited by U. Eckern, A. Schmid, W. Weber, and H. Wühl (North-Holland, Amsterdam, 1984), p. 1129.

${ }^{14}$ R. F. Miracky, J. Clarke, and R. H. Koch, Phys. Rev. Lett. 50, 856 (1983).

${ }^{15}$ D. C. Cronemeyer, C. C. Chi, A. Davidson, and N. F. Pedersen, Phys. Rev. B 31, 2667 (1985).

${ }^{16}$ M. Iansiti, Qing Hu, R. M. Westervelt, and M. Tinkham, Phys. Rev. Lett. 55, 746 (1985).

${ }^{17} \mathrm{~K}$. H. Brown, T. Bucelot, A. C. Callegari, M. M. Chen, J. H. Greiner, H. C. Jones, K. K. Kim, A. Kleinsasser, H. A. Notarys, G. Proto, B. J. Van Der Hoeven, R. H. Wang, and T. Yogi (unpublished).

${ }^{18}$ The effective noise temperature of the junction in the absence of cold attenuators is estimated to have been about $12 \mathrm{~K}$. This reduction from $300 \mathrm{~K}$ is due mainly to the severe impedance mismatch between the $50-\Omega$ cable and the $1.5-\Omega$ junction. Turning on the external noise source then elevates the effective noise temperature to about $20 \mathrm{~K}$. 